\title{
Two-dimensional dipolar Bose-Einstein condensate bright and vortex solitons on one-dimensional optical lattice
}

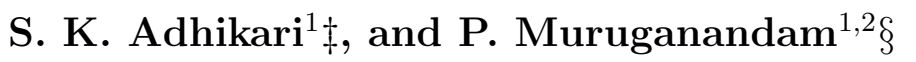 \\ ${ }^{1}$ Instituto de Física Teórica, UNESP - Universidade Estadual Paulista, 01.140-070 \\ São Paulo, São Paulo, Brazil \\ ${ }^{2}$ School of Physics, Bharathidasan University, Palkalaiperur Campus, Tiruchirappalli \\ 620024, Tamilnadu, India
}

\begin{abstract}
.
By solving the three-dimensional Gross-Pitaevskii equation we generate twodimensional axially-symmetric and anisotropic dipolar Bose-Einstein condensate bright solitons, for repulsive atomic interaction, stabilized by only a weak one-dimensional optical lattice (OL) aligned along and perpendicular, respectively, to the dipole polarization direction. In the former case vortex solitons can also be created. We show that it is possible to make a stable array of small interacting axially-symmetric dipolar solitons put on alternate OL sites. Further, we demonstrate the elastic nature of the collision of two such solitons.

PACS numbers: 03.75.Lm,03.75.Nt,05.30.Jp
\end{abstract}

‡ Email: adhikari@ift.unesp.br; URL: http://www.ift.unesp.br/users/adhikari/

$\S$ anand@cnld.bdu.ac.in 
A bright soliton is a self-reinforcing solitary wave that maintains its shape, while traveling at constant speed, due to a cancellation of nonlinear attraction and dispersive effects. Integrable solitons without any external trap or intervention for cubic nonlinearity exist only in one dimension (1D). Experimentally, bright matter-wave solitons and soliton trains were created in a quasi-1D Bose-Einstein condensate (BEC) of ${ }^{7} \mathrm{Li}$ [1, 2] and ${ }^{85} \mathrm{Rb}$ atoms [3] by turning the atomic interaction attractive from repulsive using a Feshbach resonance (FR) [4] and employing a transverse trap.

Although, the normal three-dimensional (3D) BEC soliton [5] is of great interest, such a BEC has only short-range attraction which makes it vulnerable against collapse. Physical systems are stable due to a peculiar nature of interaction among its constituents (atoms, molecules and nuclei), e.g., short-range repulsion and long-range attraction. Lately, BEC of ${ }^{52} \mathrm{Cr}$ [6, 7] and ${ }^{164} \mathrm{Dy}$ [8, 9] atoms with a large long-range dipolar interaction has been observed. Also, experimental tuning of the long-range dipolar interaction by means of rapidly rotating orienting fields [10] as well as of the shortrange atomic interaction using a FR [4] are completely under control. This engineering of the atomic and dipolar interactions makes the dipolar BEC (DBEC) an interesting system for the formation of soliton [11, 12, 13]. The long-range anisotropic dipolar interaction is attractive in some directions and repulsive in others. If it were attractive in all directions, stable robust 3D DBEC solitons, corresponding to a minimum in energy functional, would naturally be formed for repulsive short-range atomic and attractive long-range dipolar interactions [14].

Normal dipolar interaction leads to attraction along the polarization $z$ direction and repulsion along transverse directions. It is possible to have the opposite by tuning the dipole interaction to "negative" values by orienting fields [10] and this set-up was used in some studies [13, 15]. For normal dipolar interaction, an anisotropic two-dimensional (2D) soliton can be obtained for repulsive short-range atomic interaction if a weak OL is placed along $y$ axis, perpendicular to the polarization direction $z$, to overcome the dipolar repulsion in transverse directions. For dipolar interaction tuned to negative values [10], axially-symmetric 2D bright and vortex solitons can be obtained for repulsive short-range atomic interaction if a weak OL is placed along $z$ axis to overcome the dipolar repulsion in that direction in this sign-changed setting. In all cases, the dipolar repulsion is weak and we do not need any trap in other directions to stabilize a soliton. Such 2D solitons cannot be stabilized without the dipolar interaction [13].

We present a linear stability analysis for the axially-symmetric soliton [16]. We study the 2D DBEC solitons using the numerical and Lagrangian variational analysis of the 3D Gross-Pitaevskii (GP) equation. The effective Lagrangian of the variational analysis has the same structure as that of a generalized classical dynamical system with two degrees of freedom. We find that stable (referred to as "center" as it corresponds to a stable periodic orbit around a center in phase space) and unstable stationary (called "saddle" as it corresponds to a saddle point in energy) states appear and disappear through the mechanism of saddle-center bifurcation [17].

There have been studies of 2D DBEC solitons with strong harmonic traps along 
$y$ [11] or $z$ [13] axis and of 1D DBEC solitons under transverse harmonic trap [12]. The present solitons confined by only a weak OL along $y$ or $z$ axis, respectively, are distinct. The previous studies [11, 13, 15] will essentially have an approximate Gaussian density distribution along the infinite trap direction, whereas the present solitons will have an exponential density distribution due to weak finite traps in these directions. More interestingly, an OL simulates the periodic electron-atom potential in a solid and the study of solitons in an OL is also of interest in condensed-matter physics [18, 19]. We show that a new type of stable interacting 1D array of solitons can be formed in 3D space when tiny axially-symmetric interacting DBEC bright solitons are placed on alternate sites of the OL. However, if the solitons are placed on all sites of the OL, the array is destroyed due to strong long-range dipolar interaction among its constituents. Statics and dynamics of such periodic array of tiny droplets of dipolar matter are of concern in condensed matter physics [19], as they simulate many problems of general interest, such as, a periodic linear array of tiny magnets. Polarized droplets of ${ }^{52} \mathrm{Cr}$ and ${ }^{164}$ Dy have permanent magnetic dipole moment.

In a repulsive BEC on 3D OL, gap solitons having negative effective mass responsible for attraction, with the chemical potential lying in the band-gap, can be made [20, 21]. The present solitons on 1D OL, free to move in the transverse plane are bright, and not gap, solitons.

We consider a DBEC of $N$ atoms, each of mass $m$, using the GP equation: 6]

$$
i \frac{\partial \phi(\mathbf{r}, t)}{\partial t}=\left[-\frac{\nabla^{2}}{2}+V_{\mathrm{OL}}^{1 D}+g|\phi|^{2}+F\right] \phi(\mathbf{r}, t)
$$

with $g=4 \pi a N, F=\int U_{d d}\left(\mathbf{r}-\mathbf{r}^{\prime}\right)\left|\phi\left(\mathbf{r}^{\prime}, t\right)\right|^{2} d \mathbf{r}^{\prime}, \mathbf{r} \equiv\{x, y, z\} \equiv\{\rho, z\}, V_{\mathrm{OL}}^{1 D} \equiv$ $-V_{z} \cos (2 z)$ or $-V_{y} \cos (2 y)$ is the weak OL for stabilizing the soliton, $U_{d d}(\mathbf{R})=$ $g_{d d}\left(1-3 \cos ^{2} \theta\right) / R^{3}, g_{d d}=3 a_{d d} N \alpha, \mathbf{R}=\mathbf{r}-\mathbf{r}^{\prime}$, normalization $\int \phi(\mathbf{r})^{2} d \mathbf{r}=1$, a the scattering length, $\theta$ the angle between $\mathbf{R}$ and $z, a_{d d}=\mu_{0} \bar{\mu}^{2} m /\left(12 \pi \hbar^{2}\right)$ the strength of dipolar interaction, $\bar{\mu}$ the (magnetic) dipole moment of an atom, and $\mu_{0}$ the permeability of free space. The parameter $\alpha(1>\alpha>-1 / 2)$ can be tuned by a rapidly rotating magnetic field allowing the change of the sign of dipole interaction. In (1), length is measured in units of $l_{0} \equiv \lambda /(2 \pi)$, time $t$ in units of $t_{0}=m l_{0}^{2} / \hbar, V_{y}, V_{z}$, and energy in units of $2 E_{R}$, where $E_{R}=h^{2} /\left(2 m \lambda^{2}\right)$ is recoil energy, with $\lambda$ the OL wave length.

First we consider the axially-symmetric soliton for $V_{\mathrm{OL}}^{1 D}=-V_{z} \cos (2 z)$ and $\alpha<0$. In this case the Lagrangian density of (1) is [22, 23]

$$
\begin{aligned}
\mathcal{L}= & \frac{1}{2} i\left(\phi \phi_{t}^{\star}-\phi^{\star} \phi_{t}\right)+\frac{1}{2}|\nabla \phi|^{2}+2 \pi a N|\phi|^{4}+V_{\mathrm{OL}}^{1 D}|\phi|^{2} \\
& +\frac{1}{2} N|\phi|^{2} \int U_{d d}\left(\mathbf{r}-\mathbf{r}^{\prime}\right)\left|\phi\left(\mathbf{r}^{\prime}\right)\right|^{2} d \mathbf{r}^{\prime} .
\end{aligned}
$$

For a variational study we use the Gaussian ansatz [22, 23]: $\phi(\mathbf{r}, t)=\exp \left(-\rho^{2} / 2 w_{\rho}^{2}-\right.$ $\left.z^{2} / 2 w_{z}^{2}+i \gamma \rho^{2}+i \beta z^{2}\right) /\left(w_{\rho} \sqrt{w}_{z} \pi^{3 / 4}\right)$ where $w_{\rho}$ and $w_{z}$ are time-dependent widths and $\gamma$ and $\beta$ are time-dependent chirps. The effective Lagrangian $L$ (per particle) is

$$
L \equiv \int \mathcal{L} d \mathbf{r}=\left(w_{\rho}^{2} \dot{\gamma}+\frac{1}{2} w_{z}^{2} \dot{\beta}+2 w_{\rho}^{2} \gamma^{2}+w_{z}^{2} \beta^{2}\right)
$$



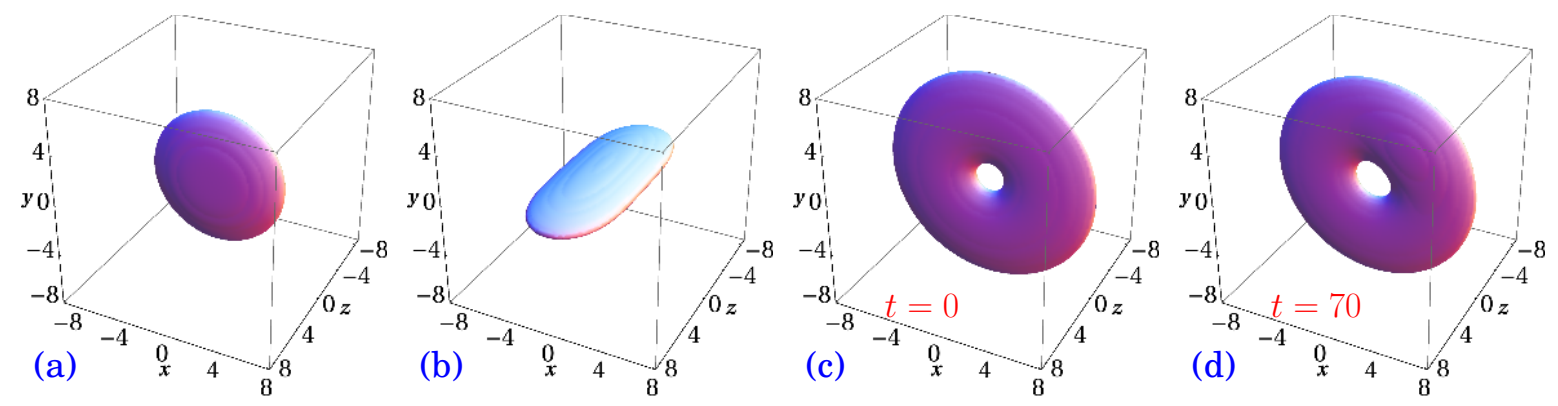

Figure 1. (Color online) (a) Numerical 3D contour of an axially-symmetric soliton for $g=50$ and $g_{d d}=-15$ on OL $V_{\mathrm{OL}}^{1 D}=-2 \cos (2 z)$. (b) The same for an anisotropic soliton with $g=50$ and $g_{d d}=20$ on OL $V_{\mathrm{OL}}^{1 D}=-2 \cos (2 y)$ and that for a vortex soliton with $g=5$ and $g_{d d}=-9$ on OL $V_{\mathrm{OL}}^{1 D}=-2 \cos (2 z)$ at $t=(\mathrm{c}) 0$ and (d) 70 . The density $|\phi(\mathbf{r})|^{2}$ on the contour is 0.001 .

$$
+E_{\text {kin }}+E_{\text {trap }}+E_{\text {int }}
$$

with kinetic, trap, and interaction energies given, respectively, by $E_{\text {kin }}=\left(1 / 2 w_{\rho}^{2}+\right.$ $\left.1 / 4 w_{z}^{2}\right), E_{\text {trap }}=-V_{z} \exp \left(-w_{z}^{2}\right), E_{\text {int }}=N\left[a-a_{d d} f(\kappa)\right] /\left(\sqrt{2 \pi} w_{\rho}^{2} w_{z}\right)$, where $f(\kappa)=$ $\left[1+2 \kappa^{2}-3 \kappa^{2} d(\kappa)\right] /\left(1-\kappa^{2}\right), d(\kappa)=\left(\operatorname{atanh} \sqrt{1-\kappa^{2}}\right) / \sqrt{1-\kappa^{2}}, \kappa=w_{\rho} / w_{z}$. The EulerLagrange equations for parameters $w_{\rho}, w_{z}, \gamma, \beta$ can be used to obtain the following equations of the widths for the dynamics of the DBEC state

$$
\begin{aligned}
& \ddot{w}_{\rho}=\frac{1}{w_{\rho}^{3}}+\frac{1}{\sqrt{2 \pi}} \frac{N}{w_{\rho}^{3} w_{z}}\left[2 a-a_{d d} e(\kappa)\right], \\
& \ddot{w}_{z}=\frac{1}{w_{z}^{3}}+\frac{1}{\sqrt{2 \pi}} \frac{2 N}{w_{\rho}^{2} w_{z}^{2}}\left[a-a_{d d} h(\kappa)\right]-\frac{4 V_{z} w_{z}}{\exp \left(w_{z}^{2}\right)},
\end{aligned}
$$

with $e(\kappa)=\left[2-7 \kappa^{2}-4 \kappa^{4}+9 \kappa^{4} d(\kappa)\right] /\left(1-\kappa^{2}\right)^{2}, h(\kappa)=\left[1+10 \kappa^{2}-2 \kappa^{4}-9 \kappa^{2} d(\kappa)\right] /\left(1-\kappa^{2}\right)^{2}$. The widths of a stationary soliton of energy $E \equiv E_{\text {kin }}+E_{\text {trap }}+E_{\text {int }}$ are obtained by solving (4) and (5) for $\ddot{w}_{\rho}=\ddot{w}_{z}=0$.

To obtain a quantized vortex of unit angular momentum $\hbar$; around $z$ axis, we introduce a phase (equal to the azimuthal angle) in wave function [24]. This procedure introduces a centrifugal term $1 /\left[2\left(x^{2}+y^{2}\right)\right]$ in the GP equation for a vortex and we adopt this method to study an axially-symmetric vortex soliton on a 1D OL along $z$ axis for the dipolar interaction tuned to negative values.

For the anisotropic $2 \mathrm{D}$ soliton on $\mathrm{OL} V_{\mathrm{OL}}^{1 D}=-V_{y} \cos (2 y)$ with $\alpha>0$, we consider a minimization of energy $E$ for a soliton using the Gaussian ansatz $\phi(\mathbf{r})=\exp \left(-x^{2} / 2 w_{x}^{2}-\right.$ $\left.y^{2} / 2 w_{y}^{2}-z^{2} / 2 w_{z}^{2}\right) /\left(\sqrt{w_{x} w_{y} w_{z}} \pi^{3 / 4}\right)$, with $E_{\text {kin }}=1 / 4 w_{x}^{2}+1 / 4 w_{y}^{2}+1 / 4 w_{z}^{2}, E_{\text {trap }}=$ $-V_{y} \exp \left(-w_{y}^{2}\right), E_{\text {int }}=\left[a+a_{d d} s\left(k_{x}, k_{y}\right)-a_{d d}\right] /\left[\sqrt{2 \pi} w_{x} w_{y} w_{z}\right]$ and

$$
s\left(k_{x}, k_{y}\right)=\int_{0}^{1} \frac{3 \kappa_{x} \kappa_{y} u^{2} d u}{\sqrt{1+\left(\kappa_{x}^{2}-1\right) u^{2}} \sqrt{1+\left(\kappa_{y}^{2}-1\right) u^{2}}},
$$

where $\kappa_{x}=w_{x} / w_{z}, \kappa_{y}=w_{y} / w_{z}$.

We perform numerical simulation of the 3D GP equation (1) using the split-step Crank-Nicolson method [25]. The dipolar term is treated by fast Fourier transformation 
Table 1. Numerical $(n)$ and variational $(v)$ energy and rms sizes $E,\langle x\rangle,\langle y\rangle,\langle z\rangle$ of bright (br) and vortex (vor) solitons. Experimental parameters $a_{d d}\left(15 a_{0}\right.$ for ${ }^{52} \mathrm{Cr}$ and $130 a_{0}$ for ${ }^{164} \mathrm{Dy}$ ), $\alpha, N$ for realizing these solitons are given for scattering length $a=5 a_{0}$ and wavelength $\lambda=10000 \AA$.

\begin{tabular}{lrrccccccc}
\hline & $g$ & $g_{d d}$ & $a_{d d}\left(a_{0}\right)$ & $\alpha$ & $N$ & $E$ & $\langle x\rangle$ & $\langle y\rangle$ & $\langle z\rangle$ \\
\hline$n$, br & 50 & -15 & 15 & -0.418 & 2400 & -0.836 & 2.00 & 2.00 & 0.450 \\
$v$, br & 50 & -15 & 130 & -0.0482 & 2400 & -0.814 & 2.086 & 2.086 & 0.423 \\
$n$, br & 50 & 20 & 15 & 0.557 & 2400 & -0.752 & 0.533 & 1.38 & 3.65 \\
$n$, vor & 5 & -9 & 130 & -0.289 & 240 & -0.744 & 4.02 & 4.02 & 0.663 \\
\hline
\end{tabular}

[22. The error of the reported numerical results is less than $1 \%$. We present in figure 1 (a) the 3D contour of the axially-symmetric bright soliton for $g=50, g_{d d}=-15$ and $V_{\mathrm{OL}}^{1 D}=-2 \cos (2 z)$. In figure 1 (b), we show the anisotropic bright soliton for $g=50$, $g_{d d}=20$ and $V_{\mathrm{OL}}^{1 D}=-2 \cos (2 y)$. For the anisotropic soliton, the numerical energy is -0.752 in agreement with the energy -0.739 obtained from the minimization in (6). The anisotropy in the $x-z$ plane in figure 1 (b) is due to dipolar interaction. In figure 1 (c) we show an axially-symmetric vortex soliton on $1 \mathrm{D}$ OL, $V_{\mathrm{OL}}^{1 D}=-2 \cos (2 z)$ for $g=5$ and $g_{d d}=-9$. A relatively large $\left|g_{d d}\right|$ is needed to overcome the centrifugal barrier and stabilize a vortex soliton. The bright solitons of figures 1 (a) and (b) are stable in real-time propagation. However, the vortex soliton with the parameters of figure 1 (c) suffers from transverse instability at large times $(t>70)$, which eventually leads to its destruction [26]. The snapshot of the vortex soliton after real-time propagation at $t=70$ in figure 1 (d) does not, however, show any distortion or sign of instability. The numerical energy and root-mean-square (rms) sizes of the solitons of figures 1 are shown in Table I with variational results in the axially-symmetric case. In this table we also show the parameters $a_{d d}, \alpha$, and $N$ for these solitons for ${ }^{52} \mathrm{Cr}$ and ${ }^{164} \mathrm{Dy}$ stabilized by a laser of wavelength $\lambda=10,000 \AA$, and atomic scattering length $a=5 a_{0}$ obtained using a Feshbach resonance.

For the axially-symmetric bright soliton, we have a conservative system with two degrees of freedom $w_{\rho}$ and $w_{z}$ with Lagrangian (3). The stable state appears and disappears by saddle-center bifurcation [17] as $V_{z}$ is increased as shown in figure 2 (a) for $g=50$ and $g_{d d}=-15$, where the unstable stationary $\left(M_{1}\right.$ and $\left.M_{2}\right)$ and stable $(S)$ states are shown in the $w_{\rho}$ versus $V_{z}$ plot. For small $V_{z}(<0.4278)$ there exists only the unstable stationary state $M_{1}$. At $V_{z}=0.4278$ and $w_{\rho} \approx 10$ a stable $(S)$ and a unstable stationary $\left(M_{2}\right)$ state appear "out of nothing" by saddle-center bifurcation. With further increase of $V_{z}$, the center $S$ comes towards the saddle $M_{1}$ and the two disappear "to nothing" by a reverse (sub-critical) saddle-center bifurcation at $V_{z}=12.2$, whereas the state $M_{2}$ moves towards infinity [17]. We show the equal-energy variational contours in the $w_{z}$ versus $w_{\rho}$ phase plot for different $V_{z}$ in figures $2(\mathrm{~b})-(\mathrm{h})$, where the positions of the stable and unstable stationary states are also shown. Figures $2(\mathrm{~d})-(\mathrm{g})$ show close ups of the appearance and disappearance of the state $\mathrm{S}$ by saddle-center bifurcations. 

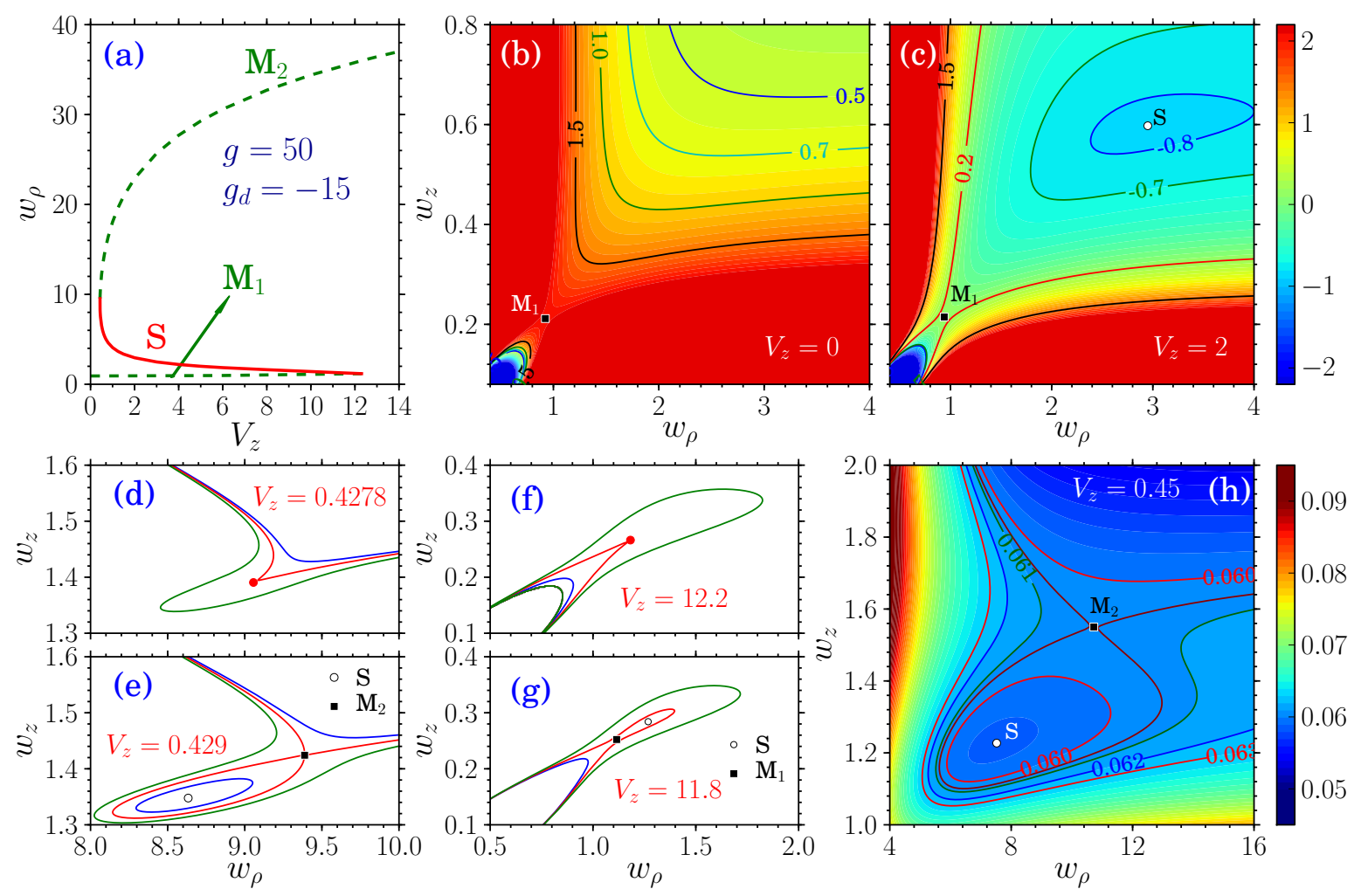

Figure 2. (Color online) (a) Bifurcation diagram showing saddle-center bifurcation involving the stable (center, $S$ ) and unstable stationary states (saddles, $M_{1}$ and $M_{2}$ ) in the $w_{\rho}$ versus $V_{z}$ plot. The lines correspond to solutions of the variational equations. The thresholds for saddle-center bifurcation are at $V_{z}=0.4278$ and 12.2. (b) - (h) The equal-energy contours in the $w_{\rho}$ versus $w_{z}$ phase plot for different $V_{z}$. A center (open circle) $S$ corresponds to a minimum of energy surrounded by closed loops in these contour plots. A saddle (solid square), $M_{1}$ or $M_{2}$, corresponds to intersection of two equal-energy lines. In $(\mathrm{d})-(\mathrm{g})$ the appearance of a saddle and a center for a small change of $V_{z}$ near the thresholds at $V_{z}=0.4278$ and 12.2 is illustrated. In all cases $g=50$ and $g_{d d}=-15$.

To perform a linear stability analysis of the axially-symmetric states, we rewrite (4) and (5) as [16]

$$
\begin{aligned}
& \dot{x}_{1}=x_{3}, \quad \dot{x}_{2}=x_{4}, \\
& \dot{x}_{3}=\frac{1}{x_{1}^{3}}+\frac{1}{\sqrt{2 \pi}} \frac{N}{x_{1}^{3} x_{2}}\left[2 a-a_{d d} e\left(\frac{x_{1}}{x_{2}}\right)\right], \\
& \dot{x}_{4}=\frac{1}{x_{2}^{3}}-\frac{4 V_{z} x_{2}}{\exp \left(x_{2}^{2}\right)}+\frac{1}{\sqrt{2 \pi}} \frac{2 N}{x_{1}^{2} x_{2}^{2}}\left[a-a_{d d} h\left(\frac{x_{1}}{x_{2}}\right)\right],
\end{aligned}
$$

where $\left(x_{1}, x_{2}, x_{3}, x_{4}\right) \equiv\left(w_{\rho}, w_{z}, \dot{w}_{\rho}, \dot{w}_{z}\right)$. These equations for widths can be written as $\dot{\mathbf{x}} \equiv \mathbf{f}(\mathbf{x})$, where $\mathbf{x} \in\left\{x_{1}, x_{2}, x_{3}, x_{4}\right\}$. If $\mathbf{x}^{(0)}$ denote the fixed points with $\dot{\mathbf{x}}=0$, so that $\mathbf{f}\left(\mathbf{x}^{(0)}\right)=0$, then the linearization matrix is $J \equiv \partial \mathbf{f}(\mathbf{x}) /\left.\partial \mathbf{x}\right|_{\mathbf{x}=\mathbf{x}^{(0)}}$ [16]. An examination of eigenvalues of $J$ reveals the nature of stability of the states. The eigenvalues come in pairs $\pm \lambda$ and lead to exponential growth unless all of them are 
imaginary corresponding to a spectrally stable equilibrium, which is of interest in the present context. For the parameters of figure 1 (a), there exist the saddle $M_{1}$ at $\mathbf{x}^{(0)} \equiv(0.9379,0.2147,0,0)$, and the center $S$ at $\mathbf{x}^{(0)} \equiv(2.9497,0.5976,0,0)$, and the saddle $M_{2}$ at $\mathbf{x}^{(0)} \equiv(22.8943,2.3485,0,0)$ of which $S$ and $M_{2}$ are shown in figure 2 (h). The eigenvalues are $( \pm 4.8644, \pm 19.9241 i)$ for $M_{1},( \pm 4.4835 i, \pm 0.2274 i)$ for $S$, and $( \pm 0.4751, \pm 0.0018 i)$ for $M_{2}$. The center with pairs of pure imaginary eigenvalues confirm its stability.

Using variational equations, we analyze the appearance of axially-symmetric bright solitons using the phase plots of $\left|g_{d d}\right|$ versus $g$ for $V_{z}=2$ in figure 3 (a). For $\left|g_{d d}\right|$ in a window of critical values, stable bright solitons can be formed. For smaller $\left|g_{d d}\right|$, there is too much repulsion and the system expands to infinity and for larger $\left|g_{d d}\right|$, there is too much attraction leading to collapse allowing only unstable stationary states. In figure 3 (b) we plot the variational and numerical rms sizes and energies for $\left|g_{d d}\right|$ corresponding to the numerical points $(\star)$ in figure 3 (a).

Next, we consider a stable soliton array by mounting tiny DBEC bright solitons along the supporting OL sites. Such solitons will be interacting due to long-range dipolar interaction. We prepare a stable array of axially-symmetric solitons each with $g=-g_{d d}=5$ by putting them on alternate sites of OL $V_{\mathrm{OL}}^{1 D}=-2 \cos (2 z)$ at $x=y=0, z= \pm(2 n+1) \pi, n=0,1,2,3$. Such an array of bright solitons with small $g$ and $\left|g_{d d}\right|$ are stable, whereas those of large $g$ and $\left|g_{d d}\right|$, e.g. the one of figure 1 (a), are unstable. In figure 4 (a) and (b) we show the initial array and the the final profile after real-time propagation at $t=200$. A similar array of anisotropic solitons has a finite life and is destroyed at large times.

Finally, we investigate the collision of two axially-symmetric bright solitons with
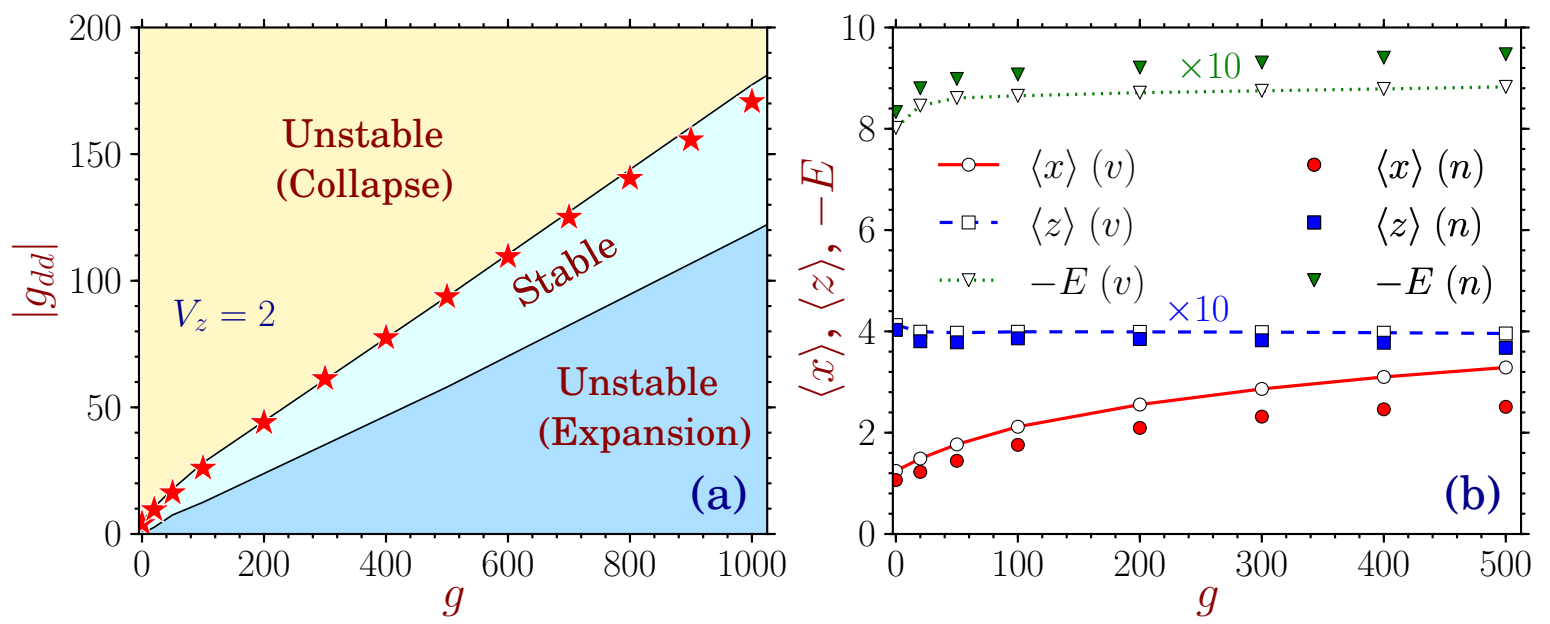

Figure 3. (Color online) (a) The phase plot of $\left|g_{d d}\right|$ versus $g$ from variational analysis of the axially-symmetric solitons showing the region of stable solitons for $V_{z}=2$. The *'s denote the numerical points showing the stable-unstable boundary. (b) The numerical (n) and variational (v) rms sizes and energy versus $g$ for $\left|g_{d d}\right|$ corresponding to the $\star$ 's in (a). 

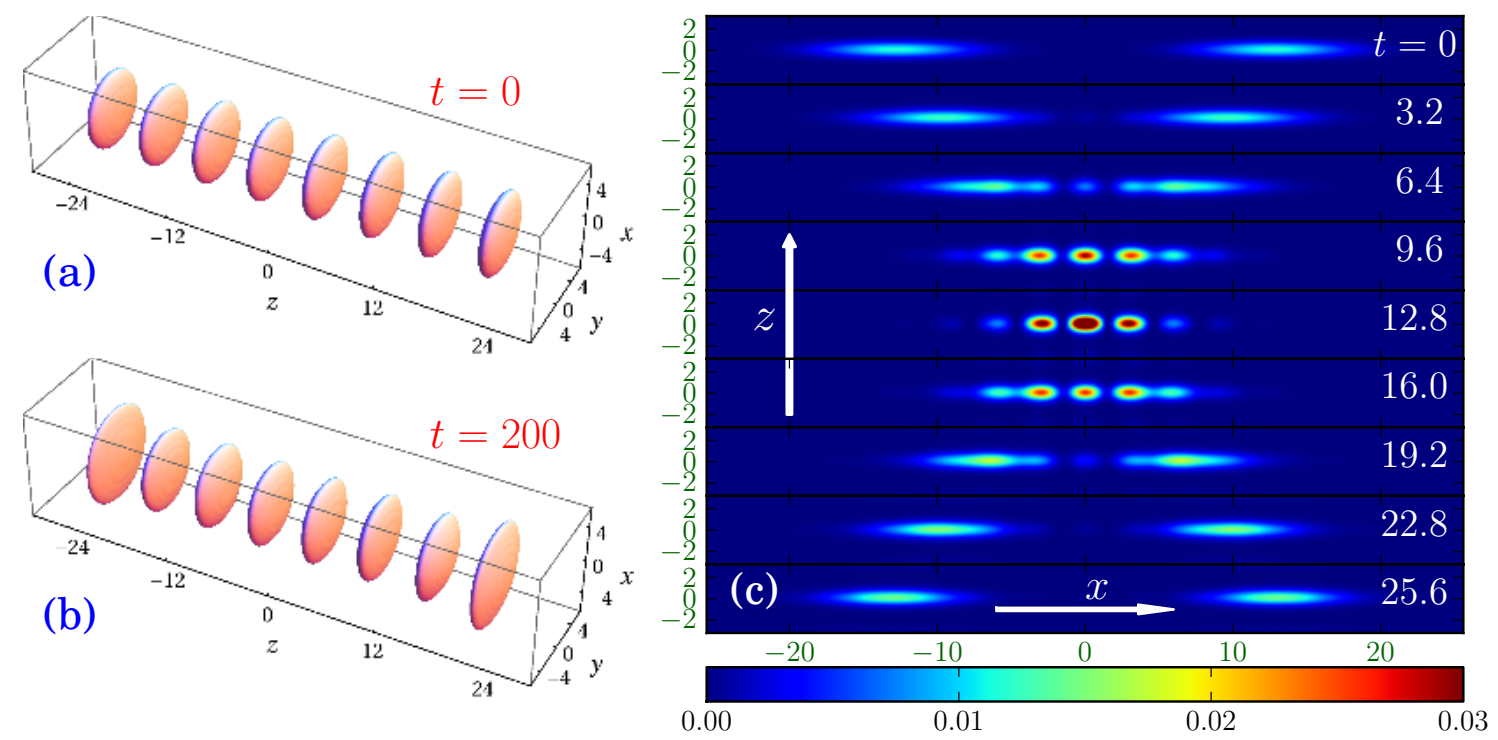

Figure 4. (Color online) 3D contour of the stable array of eight axially-symmetric solitons each with $g=-g_{d d}=5$ on OL $V_{\mathrm{OL}}^{1 D}=-2 \cos (2 z)$ at (a) $t=0$ and at (b) $t=200$. The density at contour is 0.001 . (c) Contour plot of density $|\phi(x, y, 0, t)|^{2}$ of two colliding solitons each with $g=5$ and $g_{d d}=-3$ and velocity 1 , before, during, and after collision.

$g=5$ and $g_{d d}=-3$ each, placed at $x= \pm 12.8, y=z=0$ at $t=0$. Each soliton is given a velocity of $v=1$ towards center $x=0$ by a phase factor $\exp ( \pm i x)$, respectively, in the initial wave functions. The collision dynamics is illustrated in figure 4 (c) where we show the snapshots of contour plots of density $|\phi(x, 0, z, t)|^{2}$ at different times. The solitons come towards each other, interact at $x=0$ and $t \approx 12.8$ and come out without deformation showing their robustness. In this simulation, not only the parameters of two individual solitons should lead to a stable state, the combined nonlinearities $2 g$ and $2 g_{d d}$ should also correspond to a stable configuration in figure 3 (a) to avoid collapse during collision. It was demonstrated in [13] that, under harmonic confinement, after collision at very low velocities, two quasi-2D dipolar BEC solitons may merge together to form a single soliton molecule. However, in the present case the solitons appear in a narrow window of nonlinearities $g$ and $g_{d d}$, as can be seen from figure 3 . If two equal 2D solitons, as in figure 4 (c), coalesce at low velocities, the nonlinearities of the merged soliton molecule will be outside the domain of stability in figure 3 . Hence, the formation of soliton molecule is mostly not possible in the present case. At large velocities the solitons of [13] undergo quasi-elastic collision quite similar to the present collision shown in figure 4 (c). Also, both quasi-elastic collision at large velocities and merging at low velocities of two quasi-1D solitons under transverse harmonic confinement was illustrated in [12].

To summarize, we studied different types of $2 \mathrm{D}$ bright solitons in a DBEC with repulsive atomic interaction using the solution of the 3D GP equation. Anisotropic stable 2D bright solitons in DBEC are possible on a weak 1D OL perpendicular to the 
polarization direction. Axially-symmetric stable 2D bright and vortex solitons in DBEC can be generated on a weak 1D OL along the polarization direction when the dipolar interaction is tuned to negative values [10]. In this sign-changed dipolar-interaction configuration, bright and vortex solitons are stable due to the long-range attractive dipolar interaction in the quasi-2D shape. In the axially-symmetric case, an 1D stable array of tiny solitons, placed on alternate OL sites, can be made. Such 1D array with empty OL sites between solitons is of interest in condensed matter physics [18, 19] and bears some similarity with stable checkerboard pattern of DBEC on 2D OL with empty sites in between [27], both arising due to dipolar interaction. The elastic nature of collision of two axially-symmetric solitons is also demonstrated. With present technology these stable 2D solitons and their 1D arrays can be created and studied in laboratory.

\section{Acknowledgments}

We thank Prof. B. A. Malomed for a critical reading of the manuscript and FAPESP (Brazil), CNPq (Brazil), DST (India), and CSIR (India) for partial support.

\section{References}

[1] Strecker K E, Partridge G B, Truscott A G and Hulet R G 2002 Nature 417150

[2] Khaykovich L, Schreck F, Ferrari G, Bourdel T, Cubizolles J, Carr L D, Castin Y and Salomon C 2002 Science 2561290

[3] Cornish S L et al 2006 Phys. Rev. Lett. 96170401

[4] Inouye S et al 1998 Nature 392151

[5] Perez-Garcia V M, Michinel H and Herrero H 1998 Phys. Rev. A 573837

[6] Koch T et al 2008 Nature Phys. 4218 Lahaye T et al 2007 Nature 448672 Lahaye T et al 2008 Phys. Rev. Lett. 101080401

Griesmaier A et al 2006 Phys. Rev. Lett. 97250402

[7] Lahaye T et al 2009 Rep. Prog. Phys. 72126401

[8] Lu M et al 2011 Phys. Rev. Lett. 107190401

[9] Lu M, Youn S H and Lev B L 2010 Phys. Rev. Lett. 104063001 McClelland J J and Hanssen J L 2006 Phys. Rev. Lett. 96143005

Youn S H et al 2010 Phys. Rev. A 82043425

[10] Giovanazzi S et al 2002 Phys. Rev. Lett. 89130401

[11] Tikhonenkov I, Malomed B A and Vardi A 2008 Phys. Rev. Lett. 100090406 Tikhonenkov I, Malomed B A and Vardi A 2008 Phys. Rev. A 78043614

[12] Young-S L E et al 2011 J. Phys. B 44101001

[13] Pedri P and Santos L 2005 Phys. Rev. Lett. 95200404

[14] Maucher F et al 2011 Phys. Rev. Lett. 106170401

[15] Nath R, Pedri P and Santos L 2009 Phys. Rev. Lett. 102050401

[16] See, for example, Howard J E and MacKay R S 1987 Phys. Lett. A122 331

[17] See, for example, Dullin H R and Ivanov A V 2005 Physica D 21147 Gelfreich V 2000 Physica D 136266

[18] Heinze J et al 2011 Phys. Rev. Lett. 107135303

[19] Lewenstein M et al 2007 Adv. Phys. 56243

[20] Ostrovskaya E A and Kivshar Y S 2003 Phys. Rev. Lett. 90160407 Baizakov B B et al 2002 J. Phys. B: At. Mol. Opt. Phys. 3551015 
Muruganandam P and Adhikari S K 2011 J. Phys. B: At. Mol. Opt. Phys. 44121001

[21] Eiermann B et al 2004 Phys. Rev. Lett. 92230401

[22] Góral K and Santos L 2002 Phys. Rev. A 66023613

[23] Yi S and You L 2001 Phys. Rev. A 63053607 (2001)

Yi S and You L 2004 Phys. Rev. Lett. 92193201

[24] Dalfovo F and Stringari S 1996 Phys. Rev. A 532477

[25] Muruganandam P and Adhikari S K 2009 Comput. Phys. Commun. 1801888

Adhikari S K and Muruganandam P 2002 J. Phys. B: At. Mol. Opt. Phys. 352831

Muruganandam P and Adhikari S K 2003 J. Phys. B: At. Mol. Opt. Phys. 362501

[26] Klawunn M et al 2008 Phys. Rev. Lett. 100240403

[27] Capogrosso-Sansone B et al 2010 Phys. Rev. Lett. 104125301 